\title{
CRISES SYSTÉMIQUES : EFFONDREMENT ? OU MÉTA-MORPHOSE VERS LA GRANDE TRANSITION ?
}

\author{
Author(s) / Auteur(s) : \\ Janine RENIER \\ Organis'actions \& Systèmes, Consultante en Approche Systémique des Organisations \\ Thérapeute de famille \& de couples, Formatrice à l'IEFC (Institut Européen de Formation Continue), au CFTF \\ (Centre de Formation à la Thérapie de Famille) \& au sein de réseaux psycho-médicosociaux \\ Membre de l'asbl Systèmes \& Organisations $(S \& O)$ \\ Membre de l'European Family Therapy Association (EFTA) \\ renier.janine@gmail.com
}

\begin{abstract}
Résumé :
Des systèmes complexes (Morin) et interconnectés mondialement génèrent des signes alarmants qui nous interpellent cruellement. Ils se déclinent dans de multiples crises systémiques : financière, économique, écologique (anthropocène), sociale, culturelle, démographique, migratoire, politique \& de la démocratie... Celles-ci ouvrent une période "d'incertitude et d'indécision".

Pour Morin, ce concept de crise "renvoie à une double béance : béance dans la réalité sociale elle-même où apparaît la crise, béance dans notre savoir...". Il plaide dès lors "Pour une Crisologie".

La grande transition (Narberhaus) peut-elle offrir une alternative? Elle se fonde sur une série de prémisses fortes ; un développement véritablement durable avec pour finalité le développement Humain (équité sociale) en utilisant l'économie comme moyen (économie sociale \& solidaire), tout en protégeant les écosystèmes naturels. Elle est aussi porteuse d'une valeur d'équité intergénérationnelle : "dans quel état devons-nous laisser la planète à nos enfants?"

Nous allons opérer une lecture complexe de ce "système de représentation" selon différentes écoles (complémentaires, concurrentes et antagonistes)

Wallerstein nous signale une situation structurellement chaotique aux évolutions imprévisibles; est-ce le chant du cygne de notre système historique? Au cœur de ces multiples incertitudes, s'ouvre la possibilité d'un passage vers une "formation sociale supérieure" qui alimente la thèse de la transition ; paradigme d'une trans-formation systémique, structurelle \& organisationnelle de longue durée.

Rob Hopkins initie en 2006 à Totnes au Royaume-Uni, le réseau des "villes en transition" ("initiatives de Transition"); mouvement social qui rassemble des groupes animant dans leur commune un processus de transition, lequel implique la communauté et vise à assurer la résilience (capacité à encaisser les crises économiques et/ou écologiques) face au double défi que représentent le pic pétrolier et le dérèglement climatique. Depuis, le mouvement est devenu international et compte un grand nombre d'initiatives officielles.

L'approche "multi-niveaux" (Geels-Stassart-Boulanger) offre un cadre théorique du changement systémique et permet d'étudier le rôle qu'y jouent les citoyens, les organisations de la Société civile (SMARTCSOs), les institutions économiques, culturelles \& politiques. Leurs influences réciproques se construisent lors d'un processus cyclique et itératif (co-apprentissage \& coévolution à 3 niveaux différenciés \& intégrés); micro (niches), méso (régimes), macro (paysage). Le principe de "récursion organisationnelle" est activé : multiacteurs, multi-niveaux, transinstitutionnel \& multiphases (Morin-Tremblay).

Le changement est possible quand il y a une convergence entre les processus de changement à ces trois niveaux sur le court terme, moyen terme puis long terme, ( transformation ou reconfiguration).

Les crises, chocs et pressions ouvrent des fenêtres d'opportunités vers ce changement de paradigme : transition \& Sustainable Development Goals (SDGs), alors associé à un mouvement de gouvernance polycentrique de la transition (Cassiers, Mertens), plus participatif. Un changement des "systèmes de représentations" \& des valeurs dans l'ensemble de la population permettant de se diffuser beaucoup plus rapidement dans de vastes réseaux d'influence.
\end{abstract}

\section{Keywords / Mots-clés :}

crises systémiques, systèmes-monde, grande transition, récursion organisationnelle, approche multi-niveaux, coapprentissage, co-évolution, résilience, trans-formation, reconfiguration, gouvernance participative, gouvernance polycentrique de la transition, gouvernance mondiale 


\section{ACTUALITÉ DE(S) - LA - CRISE(S)}

Des systèmes complexes (Morin, 1990) et interconnectés mondialement génèrent des signes alarmants qui nous interpellent cruellement. Ils se déclinent dans de multiples crises systémiques :

- financière, liée à une accumulation infinie de capital ;

- économique globale, façonnée par les firmes multinationales ;

- écologique, due à la surconsommation des ressources naturelles (anthropocène) ;

- sociale, face au creusement des inégalités ;

- démographique, migratoire... de sécurité ( terrorisme) ;

- politique \& de la démocratie, à l'échelle internationale ;

- humaniste, ancrée dans notre incapacité à prendre conscience de l'aventure de l'espèce humaine "mondialisée" dans une communauté de destin ;

- culturelle, traversée par les luttes identitaires et nationalistes, des luttes particularistes locales, régionales, ethniques, religieuses.

"Le développement qui se voudrait solution ignore que les sociétés occidentales sont en crise du fait même de leur développement.

Celui-ci a en effet secrété un sous-développement intellectuel, physique et moral. Intellectuel, parce que la formation disciplinaire que nous, Occidentaux, recevons, en nous apprenant à dissocier toute chose nous a fait perdre l'aptitude à relier et, du coup, celle à penser les problèmes fondamentaux et globaux. Psychique, parce que nous sommes dominés par une logique purement économique qui ne voit comme perspective politique que la croissance et le développement, et que nous sommes poussés à tout considérer en termes quantitatifs et matériels."

"Le pire est à venir de la conjonction de cinq caractéristiques majeures de la globalisation: une machine inégalitaire qui mine les tissus sociaux et attise les tensions protectrices; un chaudron qui brûle les ressources rares, encourage les politiques d'accaparement et accélère le réchauffement de la planète; une machine à inonder le monde de liquidités et à encourager l'irresponsabilité bancaire; un casino où s'expriment tous les excès du capitalisme financier; une centrifugeuse qui peut faire exploser l'Europe" (Morin, 2012)... voire le Monde.

\section{CRISES SYSTÉMIQUES ?}

Ce concept de crise est tellement usité et dans tellement de domaines différents, qu'il apparaît luimême comme étant en "crise", son extension est vaste et sa compréhension est faible :"il nous faut pourtant aller jusqu'au bout de l'opération de crisification et mettre enfin, et surtout, la notion de crise en crise." (Morin, 1976 ; Morin, 2016) Comment en effet rencontrer ce paradoxe : la crise est une réalité de plus en plus éclatante et le mot est de plus en plus utilisé, alors qu'il est "rudimentaire et creux", voire "hypnotique", tant il peine à désigner une portée explicative.

"Le problème clef est celui-ci... comment rendre éclairant le concept de crise ?"

\section{Dans la culture asiatique, en chinois mandarin (Laurent, 2012)}

"Le mot crise revêt une portée paradoxale et se dit weiji et il comprend deux caractères :

- le wei que nous retrouvons dans le mot weixian qui veut dire danger;

- et le caractère ji que nous retrouvons dans le mot jihui qui veut dire opportunité, occasion.

Il est très intéressant de voir que pour un Chinois, la crise est la concomitance du danger et de l'opportunité, une occasion au cœur du danger : celui qui ne voit que le danger passe à côté de l'opportunité sans la voir ; celui qui ne voit que l'opportunité peut prendre de gros risques." 


\section{危機}

Danger Opportunity

\section{Dans la culture occidentale : la crise au cour de la complexité}

L'intérêt pour la notion de crise a déjà été abordée et approfondie par de nombreux penseurs "Husserl, Hazard, Musil, Ricœur...", mais intéressons-nous à celle appréhendée par Edgar Morin qui revendique l'appellation de "systémique."

En lien avec la complexité, on y retrouve les éléments constitutifs de la pensée morinienne, autour de la systémique, de la cybernétique, de l'entropie/néguentropie et de la thermodynamique

En 1976, pour sa première mouture, cette élaboration théorique est réalisée après le choc pétrolier de 1973 qui signe la fin des "Trente Glorieuses". Cet événement historique critique fait émerger un besoin et une demande sociale autour "d'une science de la crise."

La temporalité est ici importante car elle est aussi contemporaine de l'apparition de la "transition" dans le contexte environnemental avec le rapport Meadows en 1972 qui insiste sur la "compréhension et la préparation en vue de la grande transition, en direction de la croissance vers l'équilibre global", en mettant en avant les risques écologiques induits par la croissance économique immodérée et démographique.

En 1987, le rapport Brundtland recommande "la transition vers un développement durable", terme qui a été et est toujours l'objet de beaucoup de controverses, nous ré aborderons ce point plus loin.

Historiquement, la notion de transition est étroitement liée à celle de développement. Pour autant, si la notion de transition est à chaque fois présente en filigrane, c'est le terme de développement durable qui est sur le devant de la scène internationale lors du Sommet de Rio en 1992, suivi par le terme d'économie verte (et équitable) lors du Sommet de Rio+20 en 2012, et qui revient avec les Objectifs de développement durable adoptés en 2015 à la COP 21 à Paris.

Les COP successives sont les conférences mondiales des parties ${ }^{1}$ à la Convention-Cadre des Nations Unies sur les Changements Climatiques (CCNUCC), de 1995 à 2017 ... et suivants

Les adeptes du concept de transition craignent une évolution du vocable les poussant à s'aligner, de manière "politiquement correcte", sur les termes publics en vogue et souvent critiqués par leur côté "green washing", le Développement Durable étant trop clairement lié à l'idée de "croissance" largement controversée.

\section{'POUR UNE CRISOLOGIE' - EDGAR MORIN}

\section{Dans l'horizon épistémologique de la complexité}

Il nous dit qu'à l'origine, la crise (Krisis en Grèce) signifie le moment où le médecin, lors d'une maladie, opère un diagnostic, il est donc synonyme de "certitude ou de quasi-certitude". Le mot a tellement dérivé qu'aujourd'hui, que la caractéristique première de la crise c'est qu'elle ouvre une période "d'incertitude et d'indécision".

Ce concept "renvoie à une double béance : béance dans la réalité sociale elle-même où apparaît la crise (Morin, 1976 ; Morin, 2016), béance dans notre savoir..."

\footnotetext{
195 pays et l'Union européenne.
} 
Ainsi, si la krisis grecque pointe un enjeu fort (moment critique de la maladie), notre époque tourmentée, ne retiendrait de cette crise que le manque, la béance : celle du savoir, mais aussi celle de la société qui peut être anéantie par la crise en question.

Edgar Morin, en liant à sa réflexion sur les crises, l'épistémologie de la "complexité" va initier chemin faisant ...une complexité théorique de la notion de crise elle-même qu'il va donc intituler: "crisologie". Le titre de son livre de 2016, réédition de l'article publié en 1976, s'intitule d'ailleurs "Pour une crisologie".

Afin de combler cette double béance, il va décortiquer les différentes composantes des crises, mais il va aussi "modéliser" "leurs interactions, leurs combinaisons, le jeu à la fois complémentaire, concurrent et antagoniste de ces processus et phénomènes, c'est à dire leur dialectisation".

\section{La complexité théorique de la crise mobilise des savoirs multiples}

Pour rendre à ce concept de crise des capacités théoriques, descriptives et interprétatives, c'est une contribution interdisciplinaire, ancrée aussi bien dans les théories des sciences de la nature que dans les sciences anthropo-sociales

Auguste Nsonsissa (2011) nous dit "Nous avons choisi de recourir à Edgar Morin, philosophe et épistémologue de notre temps, lui qui élabore, chemin faisant une crisologie, c'est-à-dire une complexité théorique de la crise [...] c'est une élaboration 'probléma-tologique' [l'expression est de Michel Meyer ${ }^{2}$ ] dans la mesure où, la théorie, selon lui, n'est pas la connaissance. Une théorie n'est ni un point d'arrivée, ni une solution, mais la possibilité de traiter un problème"

\section{La crise revêt différentes caractéristiques}

Partant des principes de thermodynamique "tout système porte en lui, puisqu'il porte de l'antagonisme, sa propre désintégration potentielle", c'est-à-dire sa ou ses crises.

$\mathrm{Au}$ premier regard, la crise se manifeste non seulement comme brisure dans un ensemble, perturbations dans un système jusqu'alors stable en apparence, mais aussi par la transformation des "complémentarités en antagonismes", le développement rapide des "déviances en tendances", l'accélération de processus déstructurant/ désintégrant (Morin)

La crise est constitué de plusieurs notions enchevêtrées (lidée de perturbation, l'accroissement des désordres et des incertitudes, le blocage/déblocage, les polémiques, les antagonismes, la manipulation des doubles contraintes, les solutions mythiques et imaginaires, etc.)

Edgar Morin dans son exposé le 16 juin 2016 sur le campus ESSEC de Cergy lors de la Journée de la Pensée Complexe, sur le thème de la "crisologie"

En résumé, dans un système vivant, on voit apparaître :

1/ des perturbations soit extérieures (par exemple : destruction des récoltes) qui déclenchent la crise soit issues de bouleversements issus de processus apparemment non perturbateurs (par exemple : croissance excessive d'une population);

$2 /$ des inhibitions (double bind) ou arrêts ou blocages des régulations que l'on peut appeler feed back négatifs ;

3/ des développements \& progressions des désordres, des puissances de ruptures, des déviances que l'on peut appeler feed back positifs ;

4/ des transformations :

- soit en quelque chose de nouveau (innovation, recherches, saut qualitatif...)

- soit dans un retour au statu quo ( feed back négatifs $\rightarrow$ homéostasie) ;

\footnotetext{
2 MEYER Michel enseigne la philosophie à l'Université libre de Bruxelles. Il a été professeur invité à la Sorbonne, à Nanterre et à Berkeley. Fondateur de la problématologie, philosophie mettant le questionnement au fondement de la pensée, il est l'auteur de nombreux ouvrages traduits dans une dizaine de langues.
} 
- soit au contraire régression vers un stade antérieur ( passéiste, mais aussi magiques, rituels, mythiques) "tout système porte en lui, puisqu'il porte de l'antagonisme, sa propre désintégration potentielle", selon les principes de thermodynamique.

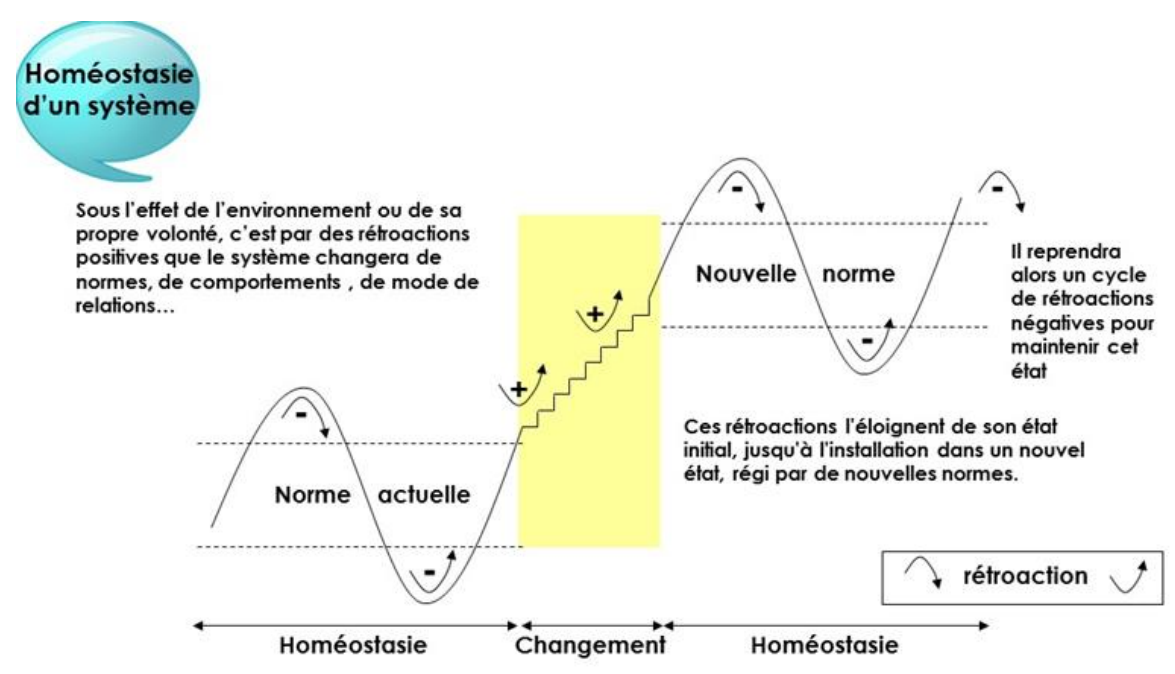

La dialectisation de toutes ces composantes permet de dégager le substrat "crisique" en tant que manifestation d'une réalité globale et complexe.

La complexité théorique de la crise va donc... "à partir de la notion d'antagonisme, [...] traverser le niveau systémique, puis le niveau cybernétique (régulation, homéostasie) puis le niveau néguentropique $^{3}$ (réorganisation permanente, développement de la complexité) des phénomènes historico-sociaux"

Dans notre période historique contemporaine, la difficulté s'accroit par le fait qu'il nous faut donc associer ces différentes crises, leurs évolutions, leurs régressions, au lieu d'en sélectionner une et éliminer les autres. Nous les vivons toutes à la fois et notre effort de connaissance doit les relier même si en même temps, chaque crise nécessite bien sûr l'étude complexe concrète de sa propre singularité.

Selon Edgar Morin, les analyses de ces crises devrait toujours faire référence à une "théorie de l'auto(géno-phéno-)éco-ré-organisation", qu'il considère comme la condition sine qua non de la possibilité d'une "théorie crisique".

Dans la perspective Morinienne, la figure de la néguentropie efface en quelque sorte les principaux caractères de rupture et de surprise de la crise, puisque celle-ci est réintégrée dans le cours inévitable des événements qui parcourt un ou plusieurs systèmes...

\section{CRISES ET MÉTA - MORPHOSE VERS LA GRANDE TRANSITION}

La "grande transition" (Narberhaus, 2011) se fonde sur une série de prémisses fortes; un développement véritablement durable avec pour finalité le développement Humain (équité sociale) en utilisant l'économie comme moyen (économie solidaire et efficace), tout en protégeant les écosystèmes naturels.

\footnotetext{
${ }^{3}$ Néguentropie: littéralement, entropie négative. Évolution d'un système des transformations d'énergie donc l'entropie diminue, contrairement au deuxième principe de la thermodynamique, soit par un apport d'énergie nouvelle, soit par un apport d'énergie organisatrice. Plus généralement : tendance à l'organisation, par opposition à l'entropie, tendance à la désorganisation.
} 
Elle est aussi porteuse d'une valeur d'équité intergénérationnelle : "dans quel état devons-nous laisser la planète à nos enfants ?"

Il s'agit du changement systémique du système global dans un processus (évolution \& ou/rupture) fondamental.

Elle peut se décliner sous différentes lectures du monde de façon complexe complémentaires, concurrentes et antagonistes.

\section{Vers un nouvel "utopistique" - Wallerstein Immanuel (2009) Modèle politique ascendant}

La situation mondiale paraît structurellement chaotique \& les évolutions sont imprévisibles, est-ce le chant du cygne de notre système historique ?

$\mathrm{Au}$ cœur de ces multiples incertitudes, s'ouvre la possibilité d'un passage vers une "formation sociale supérieure" qui alimente la thèse de l'utopistique; paradigme d'une trans-formation systémique, structurelle \& organisationnelle de longue durée.

"Nous pouvons nous représenter cette période de crise systémique comme un champ sur lequel il y a combat quant au système futur. Le résultat de cette confrontation n'est pas prévisible, mais la nature du combat et parfaitement claire. Nous sommes devant des possibilités alternatives. [...] Nous pouvons collectivement "opter" pour un nouveau système stable dont les caractéristiques fondamentales correspondent au système actuel, un système qui serait hiérarchique, exploiteur et polarisant. Sans doute cela pourrait-il se produire sous différentes formes et certaines d'entre elles pourraient-elles être encore plus dures que le système capitaliste mondial dans lequel nous avons vécu jusqu'alors. Alternativement, nous pourrions 'nous décider' en faveur d'un système radicalement différent tel qu'il n'a jamais encore existé - un système relativement démocratique et égalitaire."

Pour Wallerstein le système-monde est dans une période d'incertitude et arrivé à un point de bifurcation ${ }^{4}$ ( selon la théorie du chaos d' Ilya Prigogine).

Il s'agit d'une phase de transition vers un autre monde (ou vers d'autres mondes possibles), mais l'issue n'est pas prédictible... la complexité étant au rendez-vous...

La nouvelle utopistique devrait viser la création de formes socialisées, collectives et démocratiques de la gestion et la reproduction de la vie à l'échelle planétaire.

\section{"Villes en transition" et "initiatives de transition" Modèle a-politique ascendant}

Ici, nous allons relier les paradigmes de la crise à celui que nous avons travaillé en groupe soit celui de la "transition" et plus spécifiquement celui de "villes en transition" et "d'initiatives de transition".

Dans ces champs territoriaux locaux, le terme de transition se propage pour prendre peu à peu la place ou la suite de la notion de développement durable qui est critiqué pour sa valence économique de "croissance". Plus qu'un "en deçà" ou un "au-delà" du développement durable, il faut donc plutôt en imaginer une "seconde étape" (Theys et al., 2010).

Le réseau des "villes en transition" 5 est un mouvement social qui rassemble des groupes animant dans leur commune une initiative de transition, c'est-à-dire : "un processus impliquant la communauté et visant à assurer la résilience (capacité à encaisser les crises économiques et/ou écologiques) de la ville face au double défi que représentent le pic pétrolier et le dérèglement climatique.

Ce mouvement s'inspire d'un exercice de descente énergétique locale, effectué en 2005 par les étudiants du cours de soutenabilité appliquée de l'université de Kinsale sous la direction de Rob Hopkins (2010), formateur et enseignant en permaculture. La première mise en application a été initiée

\footnotetext{
4 Bifurcation, le mot vient du vocabulaire de la physique. Il décrit l'évolution soudaine d'un système dynamique qui connait un changement radical de trajectoire. Les systèmes complexes sont instables et évoluent par bifurcations. Loin de l'équilibre un système se caractérise par des asymétries, des turbulences et une évolution par des changements imprévisibles. Tous ses paramètres restent identiques mais une variation infime de ses conditions initiales provoque l'événement.... de la transition...

5 WIKIPEDIA, "Villes en transition", https://fr.wikipedia.org/wiki/Ville_en_transition.
} 
en 2006 dans la ville de Totnes au Royaume-Uni. Depuis, le mouvement est devenu international et compte un grand nombre d'initiatives officielles.

"Aujourd'hui, il existe plus de 4000 initiatives de Transition dans plus de 51 pays, dont la Belgique. On entend parfois l'expression 'Villes en Transition', mais on préfère aujourd'hui parler d"Initiatives de Transition' car elles naissent aussi dans des quartiers ou à la campagne. Ensemble, ces Initiatives forment un réseau mondial (le Transition Network). Elles touchent à tellement d'aspects de nos sociétés qu'on peut aussi parler de 'mouvement' " 6 .

L'originalité du mouvement des initiatives de transition par rapport aux mouvements écologistes ou sociaux existants tient en plusieurs points.

Tout d'abord, la vision de l'avenir est résolument optimiste, et les crises sont vues comme des occasions de changer radicalement la société actuelle. La deuxième originalité est que le mouvement concerne la communauté dans son ensemble car c'est cette dernière qui doit porter le changement. L'action ne doit pas exclusivement venir des gestes individuels quotidiens, ni des instances politiques via la législation.

C'est pourquoi le mouvement des initiatives de transition se considère a-partisan et choisit généralement d'éviter les confrontations (manifestations...). Ensuite, le mouvement a développé une théorie psychologique .....pour tenter de traduire le désespoir ou le déni (souvent consécutifs à la découverte du pic pétrolier et de notre dépendance au pétrole), en actions concrètes.

"L'une des grandes avancées du mouvement des Villes en Transition est sans aucun doute d'avoir su "

'débloquer' notre esprit et le tourner vers des actions positives, concrètes et libératrices. Notre meilleure chance de nous occuper efficacement du changement climatique et du pic pétrolier proviendra de notre aptitude à encourager les gens à voir dans la transition [...] une aventure dans laquelle ils peuvent investir espoir et énergie" 7

Cette vision de la transition écologique implique un rôle central des citoyens ! En effet il ne s'agit plus d'imaginer des politiques publiques en "top-down", mais en "bottom-up".

Cette originalité semble à la source du succès que connaît le mouvement des villes en transition, mais elle suscite aussi des critiques, notamment sur le manque d'engagement politique. Cette méfiance peut mener à essoufflement ou à un manque de résultats concrets à moyen terme, même si la structuration en 'transition network', d'implication mondiale gagne en forces de conviction \& en Influences

Néanmoins, étant donné le succès assez large de la notion de "transition" dans le vocabulaire politique et institutionnel, il est important de distinguer le réseau des villes en transition (un mouvement social de plus en plus coordonné) par rapport aux autres acceptions plus générales de la transition qui gagnent aussi du terrain...

\section{Gouvernance de la transition - Approche multi-niveaux Modèle hybride ascendant/descendant}

Les trois piliers de la Transition durable (sociale, économique, écologique) se chevauchent et sont interdépendants selon les principes de causalité circulaire mais aussi de "récursion organisationnelle" : multi-acteurs, multi-niveaux, trans-institutionnel \& multi-phases (Morin, 1990 ; Tremblay, 2011).

Principes clés de la gestion de la transition en tant que forme de gouvernance :

- cherche à élargir la participation en adoptant une approche multi-acteurs afin d'englober les valeurs et les croyances sociétales ;

- prend une perspective à long terme (entre 1-3 générations) en créant un panier de visions dans lesquelles des objectifs à court terme peuvent être identifiés ;

\footnotetext{
6 Réseau transition. BE, acteurs du changement positif, https://www.reseautransition.be/la-transition/

7 Servigne Pablo, citant Hopkins Rob "La transition. Histoire d'une IDEE", Publication BARRICADE, 2011.
} 
- axé sur l' apprentissage au niveau de la niche, les expériences sont utilisées pour identifier le succès d'une voie particulière et utilise le concept de "Apprendre en faisant, en faisant par l'apprentissage" ;

- une approche de la pensée systémique qui identifie les problèmes couvrant plusieurs domaines, niveaux et acteurs.

Le terme de transition existe depuis longtemps, initialement pour désigner les phases de transition des substances passant d'un état (liquide) à un autre (solide) à l'état gazeux. Il s'agit de caractériser un changement qui n'est pas linéaire mais qui est un processus chaotique. Ce modèle est appelé "l'équilibre ponctué" (Loorbach, 2007).

Ce modèle a été appliqué à l'écologie, la psychologie, des études technologiques, l'économie et la démographie.

En Hollande, c'est Rotmans, Kemps et al. (Rotmans et al., 2000 ; Rotmans et al., 2001 ; Rotmans \& Loorbach, 2001) qui ont introduit le concept de transition dans le champ du développement durable, de la gouvernance et de la politique.

Their basic hypothesis was that through the understanding of structural societal change processes (like transitions), it must be possible to formulate governance principles, methods and tools to deal with these processes (i.e. transition management).

Transitions are only one of the many pathways of change a system can pass through. We define a transition as a continuous process of societal change, whereby the structure of society (or a subsystem of society) changes fundamentally.

This societal transformation process has the following characteristics (Rotmans et al., 2000):

- it concerns large scale technological, economical, ecological, socio-cultural and institutional developments that influence and reinforce each other;

- it is a long-term process that covers at least one generation (25 years);

- there are interactions between different scale levels (niche, regime, landscape). (Loorbach, 2007) ${ }^{8}$

En Belgique, Pierre-Marie Boulanger (2008) va nous proposer la définition suivante du Management de la Transition

"Dans ce cadre, on voit apparaitre le concept de transition, définie comme un processus de transformation au cours duquel un système complexe passe d'un état d'équilibre dynamique (un régime) à un autre régime. De façon générale, cette transition résulte de l'apparition de multiples changements qui se produisent simultanément à différents niveaux et dans différents secteurs de la société (la technologie, l'économie, les institutions, les comportements, la culture, l'écologie...) et qui se renforcent et s'amplifient mutuellement jusqu'à entraîner - en cas de transition réussie - une reconfiguration globale du système considéré."

L'approche "multi-niveaux" (Stassart, 2011 ; Geels, 2002) offre un cadre théorique du changement systémique et permet d'étudier le rôle qu'y jouent les citoyens, les organisations de la Société civile (SMARTCSOs ${ }^{9}$ ), les institutions économiques, culturelles \& politiques.

Leurs influences réciproques se construisent lors d'un processus cyclique et itératif (co-apprentissage $\&$ coévolution à 3 niveaux différenciés \& intégrés).

\footnotetext{
${ }^{8}$ Leur hypothèse de base était que, grâce à la compréhension des processus structurels de changement sociétal (comme les transitions), il devrait être possible de formuler des principes de gouvernance, des méthodes et des outils pour gérer ces processus (gestion de transition). Les transitions ne sont que l'une des nombreuses voies de changement qu'un système peut traverser. Nous définissons une transition comme un processus continu de changement sociétal, par lequel la structure de la société (ou un sous-système de la société) change fondamentalement. Ce processus de transformation sociétale présente les caractéristiques suivantes (Rotmans et al., 2000): il concerne des développements technologiques, économiques, écologiques, socioculturels et institutionnels à grande échelle qui s'influencent et se renforcent mutuellement ; c'est un processus à long terme qui couvre au moins une génération ( 25 ans) ; il existe des interactions entre différents niveaux d'échelle (niche, régime, paysage). (Loorbach, 2007)

9 Narberhaus Michael et al. op. cit. http://www.smart-csos.org/
} 
Geels et Loorbach ont théorisé les interactions qui orientent les transitions en fonction de trois niveaux exerçant des pressions les uns sur les autres ( $c f$. schéma).

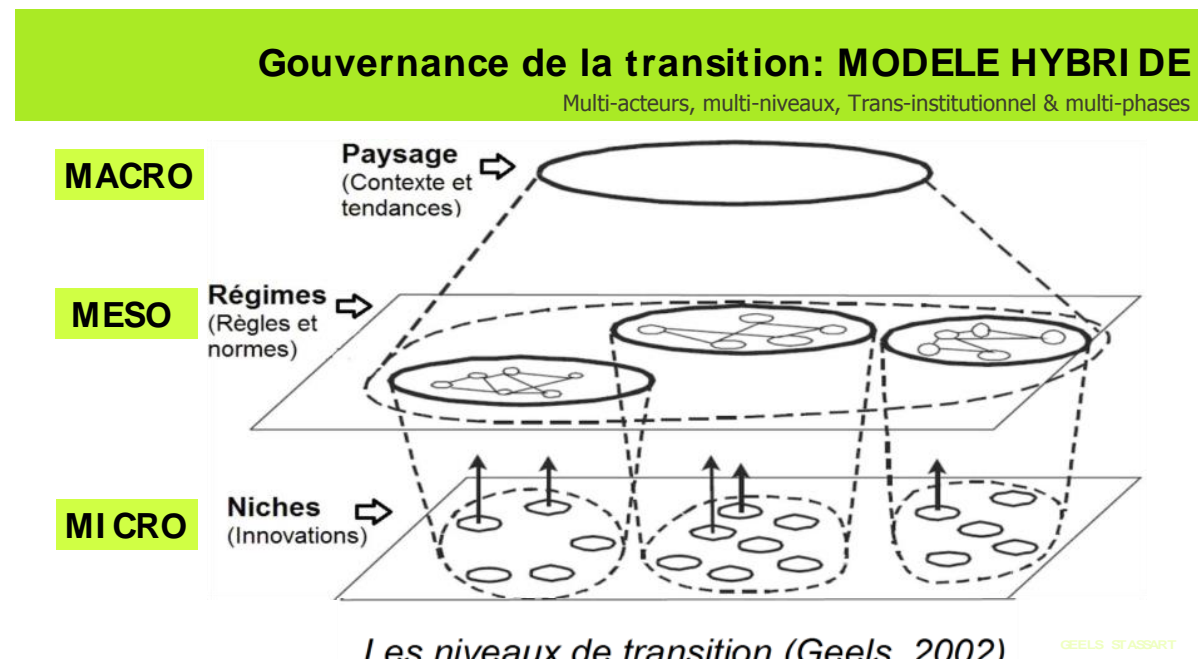

Les niveaux de transition (Geels, 2002)

\section{Micro : niches - niveau operationnel}

Les niches - se situent au premier niveau - sont le lieu d'initiatives sociales systémiques ${ }^{10}$ et d'initiatives plus radicales et d'expérimentations en marge du système établi. Elles agissent comme des environnements sûrs dans lesquels des développements révolutionnaires peuvent se développer, à l'abri du processus de sélection qui se produit au niveau du régime.

On y voit l'émergence de nouvelles idées, initiatives, technologies ou pratiques sociales, innovations sociales \& sociotechniques (mouvements locaux de transition, villes en transition, villes durables, plans de cohésion sociale, Agendas 21, mouvements convivialistes, économie sociale et solidaire, économie circulaire...). On y trouve des acteurs concernés.

Des innovations radicales peuvent y germer, de même que de nouveaux paradigmes, à l'abri de la concurrence consubstantielle au régime dominant.

Les activités opérationnelles constituent le processus d'apprentissage collectif par l'expérimentation et la mise en œuvre . Il a une durée très courte de 0 à 5 ans et se concentre sur l'innovation radicale qui transformera les "pratiques sociétales, technologiques, institutionnelles et comportementales" qui, à leur tour, filtreront et transformeront les structures, la culture et les routines du régime et peut-être influenceront le paysage.

Ces niches sont des "organisations" qui peuvent être considérées comme "un îlot de néguentropie". Cette notion de néguentropie est définie par Norbert Wiener (1962) qui trace l'image de cette capacité vivante qui s'oppose au mouvement de d'entropie générale qui va vers un état de chaos uniforme "mais tandis que l'univers comme un tout tend à se délabrer, il existe des enclaves locales dont l'évolution semble opposée à celle de l'univers en général, et dans lesquelles se manifeste une tendance limitée et temporaire à l'accroissement de l'organisation. La vie trouve refuge dans l'une de ses enclaves".

\section{Meso : regimes - niveau tactique -}

Le régime se situe au niveau méso, pris en sandwich entre le micro-niveau de la niche et le macroniveau du paysage.

\footnotetext{
${ }^{10}$ On retrouve ici la notion "d'innovations sociales systémiques qui peuvent être comprises comme de 'nouvelles solutions' (produits, services, modèles, les marchés, les processus etc.) qui répondent simultanément à un besoin social (plus efficace que les solutions existantes) et conduisent des capacités nouvelles ou améliorées et créent de nouvelles relations sociales ou collaborations. En d'autres termes, les innovations sociales sont à la fois bonnes pour la société et améliorent la capacité de la société à agir." https://www.siceurope.eu/sites/default/files/field/attachment/SIE\%20Systemic\%20Innovation\%20Report\%20\%20December\%202012_1.pdf
} 
Il constitue le mode dominant d'accomplissement d'une fonction sociale à un moment donné, fonction en lien avec le système de croyances du paysage.

- ensemble des règles et des normes, infrastructures et technologies dominantes, institutions politiques, économiques \& sociales et structures de régulation ;

- pratiques, règles et technologies dominantes qui assurent la stabilité et le renforcement des systèmes sociotechniques dominants ;

- règles et normes des systèmes sociaux qui guident les comportements, réseau d'acteurs interconnectés.

Ces ensembles assurent la stabilité du système mais également son inertie.

\section{Les activités tactiques}

Apport ascendant des niches : pour se généraliser, les innovations émergentes des niches doivent être intégrées dans le deuxième niveau, les régimes.

S'appuyant sur l'économie évolutionniste ; les régimes sociotechniques agissent comme un mécanisme de sélection et de rétention, en filtrant les échecs tout en incorporant des innovations plus 'dignes' dans le régime existant

Apport descendant du paysage: les activités tactiques se concentrent sur l'interprétation des visions créées par les activités stratégiques au niveau du régime et dans les différents réseaux, organisations et institutions impliqués.

Celles-ci viendront le plus souvent des instances politiques concernées, ou dans les entreprises du comité directeur (et de ses instances décisionnelles) soucieuses d'un développement responsable de leur utilité sociétale.

Elles chercheront à identifier les obstacles qui peuvent être rencontrés (tels que la réglementation, les conditions économiques) lors de l'interprétation de ces visions au niveau du régime.

À ce stade, les acteurs qui ont la capacité de faire des changements sont recrutés pour traduire la vision de la transition dans leurs propres agendas.

Homéostatiques, à la fois stables \& dynamiques, ils peuvent aussi présenter des résistances aux changement \& blocages. Un changement radical peut menacer potentiellement les intérêts du régime établi.

Le plus souvent, les innovations qui s'y produisent visent à optimiser le système sans le transformer globalement.

\section{Macro : paysage - niveau strategique -}

Le paysage est donc une toile du décor, réceptacle de l'interaction des acteurs au niveau du régime et de la niche.

Il désigne le contexte sociétal global qui englobe l'environnement externe et les tendances dominantes ; à la fois dans les aspects intangibles ; paradigmes, visions du monde, valeurs sociales, culturelles, politiques, écologiques, et dans l'incarnation de ces visions au travers leurs facettes tangibles; structures socioculturelles profondes, évolutions économiques et environnementales, tendances économiques, institutions, environnement bâti., bref des mouvements de fond , etc...

Les activités stratégiques conduiront à des changements dans la "culture" du système sociétal au niveau du paysage. C'est ce niveau stratégique du Management de Transition qui vise un changement de paradigme. Il devrait autoriser l'augmentation de la conscience environnementale. Mais pas seulement, notre vision du monde devrait se méta-morphoser autour du développement Humain et d'une co-construction de l'universel.

Les gouvernements (convaincus) vont créer une pression descendante par les législations et la réglementation, l'utilisation des incitations du marché tout en encourageant le "processus d'apprentissage collectif" en soutenant financièrement l'innovation et en donnant accès aux informations. 


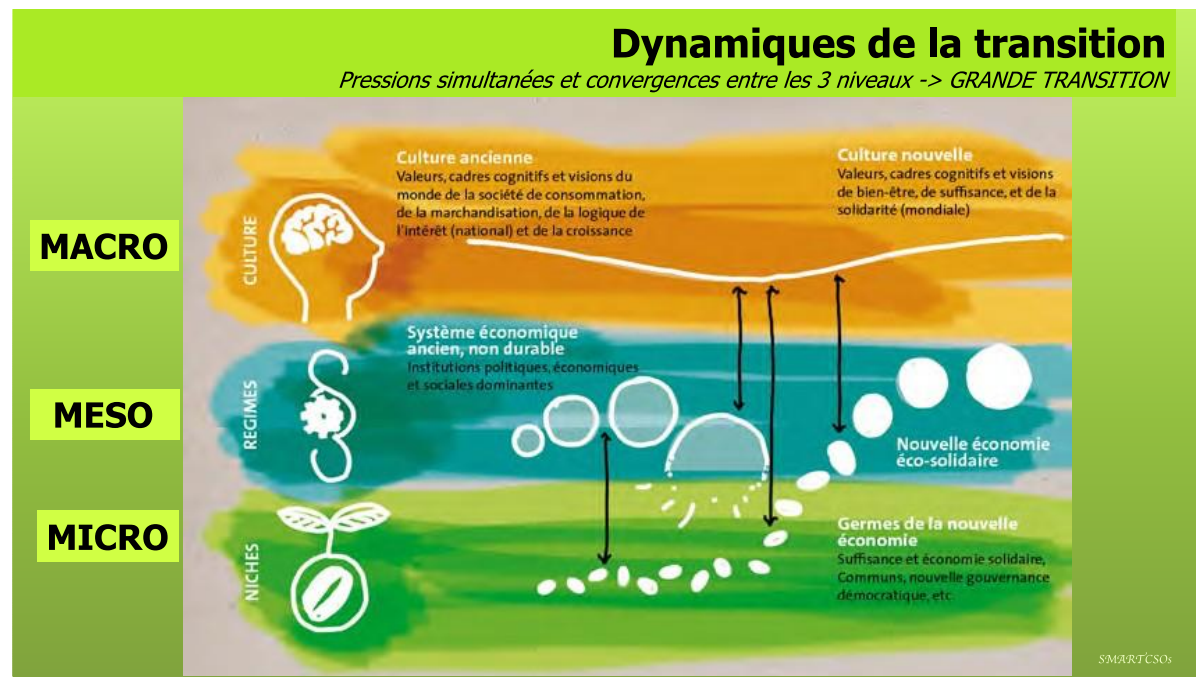

La finalité de cette activité est à l'échelle du long terme (30 ans / 3 générations) et englobe donc le processus de développement de la vision; l'action collective d'établissement de buts et de normes par la discussion et la formulation d'objectifs à long terme.

Il ne peut pas être modifié facilement, car il ne subit pas l'influence directe des acteurs, les crises le "secoue" pourtant ardemment!!

\section{Double stratégie - version radicalité}

Double strategie pour les alternatives, dans la logique du reformisme revolutionnaire pour une "revolution évolutive" qui altere fondamentalement les régimes existants

Ce sont les pressions exercées simultanément par ces trois niveaux qui peuvent entraîner des transitions, le changement est possible quand il y a une convergence entre les processus de changement aux trois niveaux (Paysage - Régimes - Niches) sur le court terme, moyen terme puis long terme.

"La transformation consiste en un cumul d'ajustements et de réorientations. Les innovations issues des niches, insuffisamment développées encore, sont adoptées sur le mode symbiotique ( qui n'exclut cependant pas des actions directes telles que le boycott) pour résoudre des problèmes rencontrés par le régime dominant sans modification de l'architecture de base de celui- ci.

Par contre, dans la reconfiguration, les innovations, après une entrée similaire dans le régime dominant, entraînent ensuite des changements profonds dans cette architecture de base ; changements qui peuvent à leur tour faciliter l'adoption de nouvelles innovations."

Le vrai but de la grande transition est de faire bouger les différents niveaux, par isomorphisme et d'aller vraiment vers ce que Edgar Morin appelle la méta-morphose, puisqu'il y a une reconfiguration de la forme du système

Dans un modèle de changement systémique, la transition est la période pendant laquelle un système déstructuré et en rupture de sens voit les transformations majeures se réaliser dans l'ensemble de ses sous-systèmes, jusqu'à provoquer la reconfiguration de l'ensemble du système lui-même.

Cette phase de transition est suivie d'une étape finale de recherche et de recouvrement d'un nouvel équilibre (que l'on peut appeler harmonie ou nouvelle homéostasie) au cours de laquelle la mutation peut devenir irréversible. 


\section{Réseau et militance}

Au niveau macro, un changement des "systèmes de représentations" et des valeurs viendrait solidifier le soutien des citoyens aux innovations radicales, leur permettant de se diffuser beaucoup plus rapidement dans de vastes réseaux d'influence (Wheatley \& Frieze, 2006).

\section{Quel rôle pour les pouvoirs publics ? Version consensus au niveau local, regional, national}

\section{Accompagnement de la transition écologique un enjeu de justice sociale et de démocratie}

Il n'y a pas diverses transitions (énergétique, sociale, agro-écologique, démocratique, politique.. etc.) mais une Transition dotée de multiples visages à aborder dans leur complexité multidimensionnelle. Le besoin de changement parait 'urgentissime' mais il nécessite un changement de modèle et non une série de mesures juxtaposées qui nécessiterait donc une intervention politique ....plus au niveau méta! Dans la sphère politique, nous avons donc parlé de 'gouvernance de la transition', il s'agit donc d'accompagner ces mouvements citoyens spontanés sans qu'ils tournent le dos aux institutions, en évitant néanmoins le risque de récupération politique. Il faudrait donc se centrer d'avantage sur 'l'accompagnement' de ces différents processus de transition. Ceci dans la lignée de la convention d'AARHUS $^{11}$

Voire même au plan politique, une réelle remise en forme de législations permettra de nouvelles initiatives :

"[...] qu'elle soit écologique, énergétique, sociale, solidaire, économique, démocratique, numérique ou encore managériale, la transition se caractérise par une transformation profonde des systèmes. Une pluralité d'acteurs se revendique du concept de transition : la recherche s'attelle à en identifier les ressorts, les institutions souhaitent en dessiner les orientations et la société civile s'engage et l'aiguillonne à travers des expérimentations innovantes" (Boissonade, 2017).

"Si les transitions systémiques ne peuvent être complètement contrôlées, il est possible de les faciliter et de les orienter. À ce titre, les institutions ont un rôle clé, d'une part en identifiant et en encourageant les initiatives pionnières vertueuses (valorisation, soutien financier ou technique, etc.), d'autre part en encourageant et en accompagnant le changement d'échelle pour aller vers une mise en mouvement globale de la société (évolution des cadres législatifs et réglementaires, définition de cadres stratégiques de long terme, inflexion des politiques publiques, etc.) et enfin en anticipant et en appréhendant les grandes évolutions futures pour en réguler les effets et en relever au mieux les défis." 12

Associé à un mouvement Ascendant/descendant, la gouvernance de la transition (Cassiers, Maréchal, Méda, 2017), plus participatif, requérant l'exercice d'un pouvoir plus polycentrique (Bauwens \& Mertens, 2017) notamment à destination des économies sociales et solidaires

Une vision écosystémique ne peut dépendre que d'une dynamique représentative \& d'empowerment. Elle se doit de prendre en compte l'ensemble des composantes sociales, politiques et économiques, dans le cadre d'une dynamique participative ; dynamique non seulement ouverte à tous, mais plus largement inclusive. La cohésion sociale nous semble ainsi primordiale. ( Renier, 2016)

\footnotetext{
${ }^{11}$ LA CONVENTION D'AARHUS sur l'accès à l'information, la participation du public au processus décisionnel et l'accès à la justice en matière d'environnement, signée le 25 juin 1998 par trente-neuf États (ratification par la Belgique le 21/01/2003) est un accord international visant la "démocratie environnementale". Ses trois grands objectifs sont ; - améliorer l'information environnementale délivrée par les autorités publiques, vis-à-vis des principales données environnementales ; - favoriser la participation du public à la prise de décisions ayant des incidences sur l'environnement (par exemple, sous la forme d'enquêtes publiques) ; - étendre les conditions d'accès à la justice en matière de législation environnementale et d'accès à l'information.

${ }^{12}$ Commissariat général au développement durable, "la transition, analyse d'un concept", juin 2017, France.
} 


\section{L'appropriation de la transition par les institutions nationales et internationales, une approche circulaire et recursive}

Historiquement, la notion de transition est étroitement liée à celle de développement. Pour autant, si la notion de transition est à chaque fois présente en filigrane, c'est le terme de développement durable qui est sur le devant de la scène internationale lors des différents sommets mondiaux.

L'ONU s'est dotée en 1992, à l'occasion du sommet de la Terre de Rio de Janeiro, d'un cadre d'action de lutte contre le réchauffement climatique: la Convention-Cadre des Nations Unies sur les Changements Climatiques (CCNUCC). Cette convention réunit presque tous les pays du monde qui sont qualifiés de "Parties". ${ }^{13}$ Leurs représentants se rassemblent une fois par an depuis 1995, lors des "COP" (Conferences of the Parties)

Lors du Sommet de Rio en 1992, se développe le terme de "développement durable", suivi par le terme "d'économie verte (et équitable)" lors du Sommet de Rio+20 en 2012, et qui revient avec les Objectifs de "développement durable" \& de "transition écologique" adoptés en 2015 lors de la COP 21, laquelle s'est tenue à Paris et a permis de conclure un accord engageant 195 États à réduire leurs émissions de gaz à effet de serre.

Très récemment en septembre 2018, s'est tenue à San Francisco, une "COP des acteurs non étatiques" contre le réchauffement climatique ; villes, régions, entreprises et $O N G$ veulent aiguillonner les États lors de la COP24 qui se déroulera à Katowice en Pologne en décembre 2018 . Ceci à un moment où le retrait américain de l'accord de Paris a ralenti les ardeurs des cosignataires à remplir les objectifs affichés aux Nations unies.

Nous voyons bien ici à nouveau la pertinence de ce processus récursif et circulaire descendantascendant-descendant-ascendant... entre politique et société civile pour tenter de provoquer des pressions et des chocs de manière simultanée aux différents niveaux.

Les adeptes du concept de transition craignent toujours dans ce cadre formel et "institutionnel" une évolution du vocable les poussant à s'aligner, de manière "politiquement correcte", sur les termes publics en vogue et souvent critiqués par leur côté "green washing", le Développement Durable étant toujours clairement lié à l'idée de "croissance" largement controversée.

\section{Transition \& sustainable development goals}

\section{Approche internationale incontournable pour une onu supranationale ??}

"Il est l'heure de changer de civilisation" et de modeler la "Terre patrie" nous dit encore Edgar Morin qui situe la crise au cœur de notre temps.

Il faut atteindre co-construire une vision de l'universel ; cela suppose un dialogue entre points de vue et modèles culturels, politiques et éthiques différentes, entre stabilisations, progressions et régressions....

Les défis mondiaux tels que la guerre, le changement climatique, la pauvreté et les inégalités sont des défis qui envahissent les États-nations et les institutions internationales d'aujourd'hui. Faire ce qu'il faut nécessite plus que d'avoir les bonnes politiques; il faut disposer des structures politiques appropriées pour les mettre en œuvre.

Dans une récente enquête commandée par la Global Challenges Foundation ${ }^{14}$ couvrant l'Australie, le Brésil, la Chine, l'Allemagne, l'Inde, la Russie, l'Afrique du Sud, la Suède, le Royaume-Uni et les États-Unis, $82 \%$ en moyenne des répondants ont convenu que l'ONU doit être réformée pour mieux faire face aux risques mondiaux actuels et futurs. En outre, sept répondants sur dix étaient favorables à l'idée de créer une nouvelle organisation supranationale " pour prendre des décisions mondiales exécutoires pour faire face aux risques mondiaux ".

\footnotetext{
13195 pays et l'Union européenne.

${ }^{14}$ Lisez le résumé du sondage ici : https://www.democracywithoutborders.org/6017/.
} 
Malgré la montée du populisme nationaliste, ces chiffres montrent qu'une grande partie de la population mondiale croit au renforcement de la collaboration internationale, même en dotant un organisme mondial de pouvoirs supranationaux. Il va sans dire qu'un tel organe devrait être démocratique et représentatif.

Au niveau international, les crises, chocs et pressions y ont ainsi ouvert des fenêtres d'opportunités vers ce changement de paradigme dans la gouvernance Mondiale: transition \& Sustainable Development Goals $\left(\mathrm{SDGs}^{15}\right)$, on voit donc apparaître les deux mots reliés.

Pour parvenir à une civilisation mondiale pacifique, juste et durable, il faut faire un bond en avant vers un gouvernement mondial fédéral.

La création d'un parlement mondial démocratique serait la pièce maîtresse de ce projet. Le livre de Jo Leinen et d'Andreas Bummel (2018) décrit la pertinence actuelle de la mise en œuvre future de cette idée monumentale.
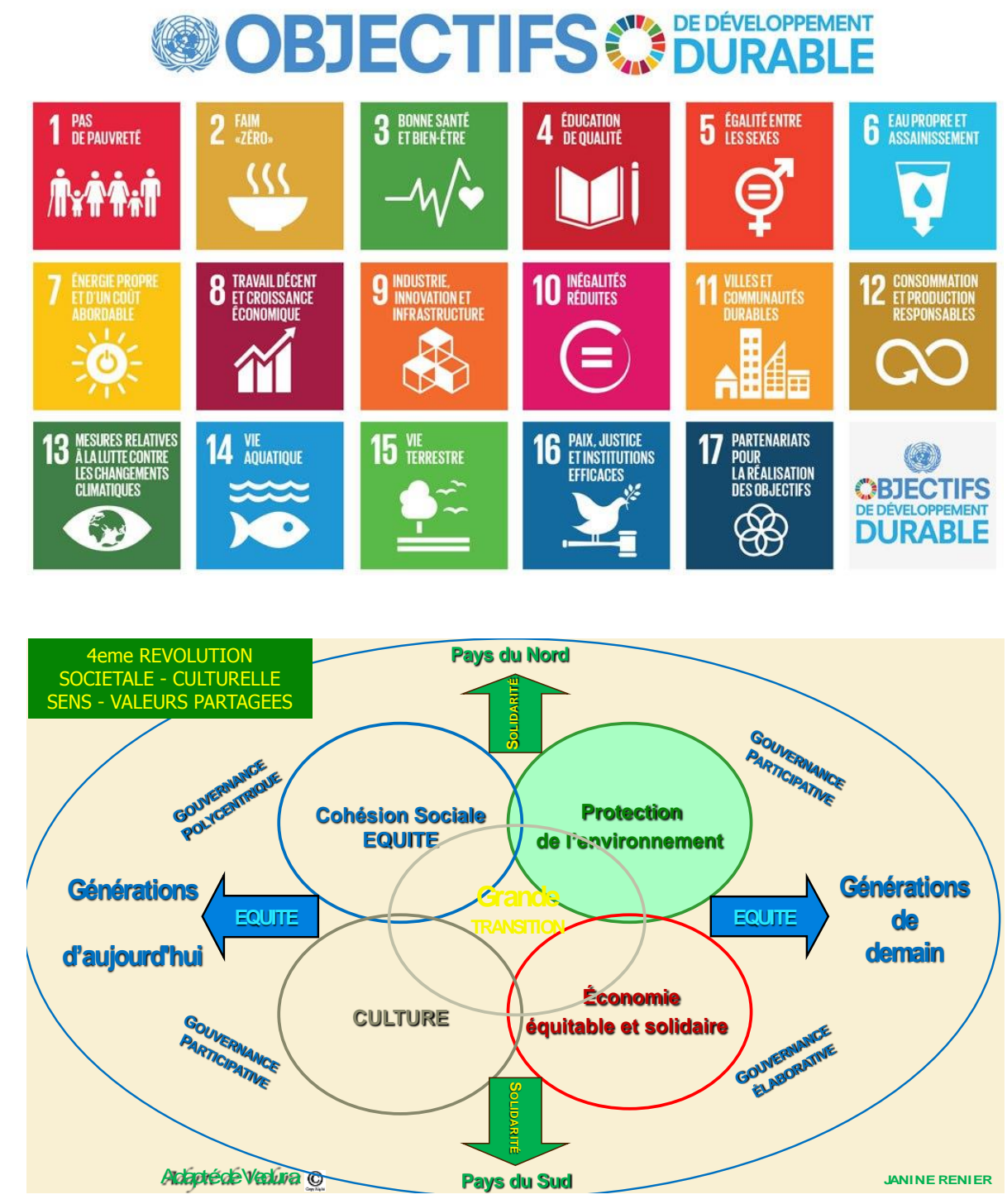
1517 Sustainable Development Goals (SDGs), United Nations, https://www.un.org/sustainabledevelopment/sustainable-
development-goals/. 


\section{RÉFÉRENCES}

BAUWENS, Thomas \& MERTENS, Sybille (2017). "Économie sociale et gouvernance polycentrique de la transition", pp.89-121.

BOUILLOUD, Jean-Philippe (2012). "De la crisologie à la risquologie", Communications 2012/2 (n 91), p. 153-159.

BOISSONADE, Léa (2017). "La transition, Analyse d'un concept", Commissariat général au développement durable, Ministère de la transition écologique et solidaire, France.

BOULANGER, Pierre-Marie (2008). "Une gouvernance du changement sociétal : Le transition management", Numéro 11, La Revue Nouvelle, Belgique.

CASSIERS Isabelle, MARÉCHAL Kevin \& MÉDA Dominique (2017). Vers une société post-croissance, Éditions de l'Aube, 224pp.

CASSIMAN Stéphane, Le Management de Transition vers la Soutenabilité. Aperçu de la théorie et de quelques critiques. Mémoire de fin d'études 2007-2008. IGEAT - ULB.

GEELS, F.W. (2002). "Technological transitions as evolutionary reconfiguration processes: A multi-level perspective and a case study". Research Policy. 31, 1257-1274.

HOPKINS, Rob (2010). Manuel de Transition, Silence/Ecosociété.

LAURENT, Philippe (2012). "La crise, une opportunité au cœur du danger". L'express emploi.

LEINEN Jo \& BUMMEL Andreas (2018). A World Parliament Governance and Democracy in the 21st Century, Avril 2018, https://www.democracywithoutborders.org/world-parliament-book/.

LOORBACH, D. (2007). Transition management. New mode of governance for sustainable development, Utrecht: International Books.

MEADOWS Donella H., MEADOWS Dennis L., RANDERS Jørgen, William W. BEHRENS III (1972). The Limits to Growth, A report of the Club of Rome's Project on the Predicament of Mankind, p. 24, New York, Universe Books.

MORIN, Edgar (2016). "Sans tabou", débat entre Edgar Morin et François L'Yvonnet sur le carnet de l'Herne, "Pour une Crisologie", 16/06/16, https://www.youtube.com/watch?v=pw5RoVDCCfE.

MORIN, Edgar (2016). Pour une crisologie, Paris, carnets de l'Herne.

MORIN, Edgar (2012). La Voie : Pour l'avenir de l'humanité, Poche.

MORIN, Edgar (1990). Introduction à la pensée complexe, Paris, E.S.F., collection communication et complexité.

MORIN, Edgar (1976). "Pour une crisologie", Communications, n² 25, pp.149-163, https://www.persee.fr/doc/comm_0588-8018_1976_num_25_1_1388

NARBERHAUS, Michael en collaboration avec Christina Ashford, Maike Buhr, Friederike Hanisch, Kerem Şengün et Burcu Tunçe (2011). Réussir la grande transition, Cinq leviers de changement pour les organisations de la société civile, http://www.smart-csos.org/

NSONSISSA, Auguste (2011). "Pour une crisologie". Université Marien Ngouabi - Brazzaville, hermes 60, pp.135-144.

RENIER, Janine (2016). "Vertiges et promesses de la complexité... quelles méta-morphoses pour nos démocraties désenchantées ?".Acta Europeana Systemica - Vol. 6.

http://aes.ues-eus.eu/aes2016/15_Renier-Janine_FR.pdf

RENIER, Janine (2018). Avances Sistémicos, Año 1, Número 2 -2 2a Parte, pp.40-42, juin.

STASSART, Pierre M. (2011). "Introduction à la théorie de la transition", APERe 20 ans, 12 mai, http://www.apere.org/doc/110512 Pierre Stassart Ulg1.pdf

TREMBLAY, Louise (2011). Gouvernance des transitions vers la durabilité, Centre Universitaire de Formation en Environnement, Université de Sherbrooke, Gatineau, Québec, Canada, 18 octobre.

WALLERSTEIN, Immanuel (2009). Comprendre le monde - Introduction à l'analyse des systèmes-monde - La Découverte /Poche.

WHEATLEY, M. \& FRIEZE, D. (2006). Using emergence to take social innovation to scale.

WIENER Norbert (1962). Cybernétique et société, UGE, (10/18) Paris. 
